\title{
SYNTHESIS AND CHARACTERIZATION OF BENZOIC ACID COMPLEXES WITH BIOLOGICALLY ACTIVE METAL IONS
}

\author{
M.S. Selvakumar* and S.R. Bheeter**
}

\begin{abstract}
Animal and plants contain carboxylic acids, which are involved in their metabolic reactions. It is also well known that metal ions are involved in their metabolic reactions. So far the complexation of carboxylate ions with metal ions has been extensively studied. Since neutral or acidic medium is existing in most of the biological reactions in animals and plants it is interested to study the complexation of organic carboxylic acids in neutral medium with biologically active metal ions such as manganese, cobalt, nickel, copper and zinc. Complexes of benzoic acid were prepared in neutral medium and were characterised. In such complexes the carboxylic acid group is notionised and carbonyl oxygen of the carboxylic acids are coordinated to the metal ions. However the microbial study is not the scope of this work.
\end{abstract}

\section{Introduction}

Biologically important metals $\mathrm{Mn}, \mathrm{Co}, \mathrm{Ni}, \mathrm{Cu}$ and $\mathrm{Zn}$ were taken for this study. Manganese is an essential component of the photosynthetic mechanism of plants. ${ }^{\prime}$

\footnotetext{
* Reader in Chemistry, St. Joseph's College (Autonomous), Trichy - 620002

** Professor in Chemistry, St. Joseph's College (Autonomous), Trichy - 620002
} 
$V$ it $B_{12}$ is an important complex of cobalt: ${ }^{2}$ Nickel is a component of the enzyme urease. ${ }^{3}$ Copper is cofactor of a number of oxidation enzymes. ${ }^{4}$ Zinc is found in the enzyme like DNA polymerase, carbonic anhydrase and alcoholic dehydrogenates. ${ }^{5}$ These metals and the ligand benzoic acid were taken and complexes were prepared in the methanol solvent.

\section{Preparation and Purification}

Metal chloride and benzoic acid were taken in the molar ratio 1:6. They were dissolved in methanol and refluxed for six hours. The complexes were purified by washing with ether.

\section{Analysis of the Complexes}

The percentage of metal ions in the complexes was experimeritally found by volumetric methods. ${ }^{6}$ The experimentally determined percentage of metal ions is analogous with the percentage of metal ions of the proposed geometry.

\section{Electrical Conductivity}

The experimentally determined molar conductivity of complexes in acetonitrile shows that benzoic acid complexes are of 1:0 type electrolytes. The presence of chloride ion in the benzoic acid complexes is evident only after the decomposition of the complexes, due to their presence in the coordination sphere.

\section{Magnetic Moments}

Manganese (II) complexes are high spin complexes with spin only magnetic moment 5.9 B.M. The experimentally determined room temperature magnetic moment of benzoic acid manganese (II) chloride complex is 5.93 B.M. suggesting octahedral or tetrahedral geometry. However, the metal ion percentage calculation suggests that the complex is octahedral in geometry.

Co (II) has the spin only magnetic moment 3.89 B.M. The room temperature magnetic moments for octahedral complexes are in the range of 4.7-5.2 B.M. due to orbital contribution and that for tetrahedral complexes are in the range of $4.4-4.8$ B.M. The experimentally determined magnetic moment of benzoic acid 
cobalt(ll) chloride complex is 5.2 B.M. which suggest that the complex is octahedral in geometry.

The spin only magnetic moment of $\mathrm{Ni}(I)$ is 2.83 B.M. Octahedral Ni(II) complexes do not have much orbital contribution. Hence the magnetic moments are in the range of $2.83-3.1$ B.M. In tetrahedral field, due to orbital contribution the magnetic moments are in the range of $3.1-3.5 \mathrm{B.M} .{ }^{7}$ The synthesised benzoic acid nickel (II) chloride complex has the magnetic moment 2.84 B.M. which suggest octahedral geometry for the complex.

Octahedral or tetrahedral geometry is expected to have little effect on the magnetic moment of copper (II) complexes. With spin orbit coupling it has the magnetic moment about 1.9 B.M. Benzoic acid copper(il) chloride complex has the magnetic moment 1.94 B.M. suggesting octahedral geometry.

As expected, the zinc (II) chloride complex of benzoic acid is diamagnetic in nature.

\section{Infra-red Spectrum}

The presence of anion and the absence of symmetric and asymmetric $\mathrm{C}=\mathrm{O}$ stretching frequency in the spectra of complexes suggest the carboxylic acid is not ionised in the complexes. That is, the complexes are not the salts of carboxylate.

The shift in $\mathrm{C}=\mathrm{O}$ stretching to lower region in the spectra of complexes suggest that the carbonyl oxygen of the unionised carboxylic acid is coordinate to the metal ion.

Vandoore and Hannik reported that the unionised acetic acid is coordinated to metal atom through the carbonyl oxygen. The shift in the carbonyl stretching frequency of the carboxylic acid group to the lower frequency suggest the coordination of carbonyl oxygen to metal analogous to the complexes of unionised acetic acid. ${ }^{8}$

The C-O and O-H deformation at 1250-1350 $\mathrm{cm}^{-1}$ is slightly shifted in the spectra of complexes in comparison with that of ligand. The other frequencies including $C=C$ of the aromatic ring at $1500-1600 \mathrm{~cm}^{-1}$ are not very much affected in the spectra of complexes.

\section{Electronic Spectrum}

The $\mathrm{S}^{6}$ ground state of the free $\mathrm{Mn}(\mathrm{II})$ ion is not split by the ligand field. All excited states of the $d^{5}$ system have different spin multiplicity from the ground state and 
transition to them are spin forbidden. Due to very weak absorption we could not get any appreciable peak in the electronic spectra of $\mathrm{Mn}(\mathrm{Il})$ complexes. However, pale pink colour and analytical data suggest octahedral geometry.

The ground state term of $\mathrm{Co}(\mathrm{Ill})$ ion is ${ }^{4} \mathrm{~T}$, which arised from the $\mathrm{t}_{29}{ }^{5} \mathrm{e}_{\mathrm{g}}{ }^{2}$ configuration. An octahedrally coordinated cobalt (ii) ion should have three spin allowed d-d transition. ${ }^{4} \mathrm{~T}_{19}(\mathrm{~F}) \rightarrow{ }^{4} \mathrm{~T}_{2 g}(\mathrm{~F}),{ }^{4} \mathrm{~T} \mathrm{~T}_{18}(\mathrm{~F}) \rightarrow{ }^{4} \mathrm{~A}_{2 g}(\mathrm{~F})$ and ${ }^{4} \mathrm{~T}_{1 g}(\mathrm{~F}) \rightarrow{ }^{4} \mathrm{~T}_{1 g}(\mathrm{P})$. The transitions ${ }^{4} T_{19}(F) \rightarrow{ }^{4} T_{2 g}^{2 g}(F)$ and ${ }^{4} T_{1 g}(F) \rightarrow{ }^{4} A_{2 g}(F)$ are not observed as the first one is weak transition and the second one is a two electron process. The band in the visible region near $20000 \mathrm{~cm}^{-1}$ is assigned to the ${ }^{4} \mathrm{~T}_{1 g}(\mathrm{~F}) \rightarrow{ }^{4} \mathrm{~T}_{1 \mathrm{~g}}(\mathrm{P})$ transition. Similarly for the tetrahedral complexes, the lower energy band due to ${ }^{4} \mathrm{~A}_{2} \rightarrow{ }^{4} \mathrm{~T}$, lies in the infra-red region where it is frequently overlapped by vibrational levels and so this band is not often observed. The ${ }^{4} A_{2} \rightarrow{ }^{4} T_{1}(F)$ and ${ }^{4} A_{2} \rightarrow{ }^{4} T_{1}(P)$ band lie in the near infra red and visible regions respectively. They differ most significantly from the octahedral absorption in their much greater intensities, thus allowing ready distinction between the two stereo chemistries. In the present study the benzoic acid cobalt (II) chloride complex shows electronic spectra of absorption at 18860 $\mathrm{cm}^{-1}$ which is due to the transition ${ }^{4} \mathrm{~T}_{19}(\mathrm{~F}) \rightarrow{ }^{4} \mathrm{~T}_{19}(\mathrm{P})$ in an octahedral environment. Hence it is very clear that the synthesised complexes are octahedral in geometry.

For octahedral $\mathrm{Ni}(\mathrm{II})$ complexes the transitions and their bands are ${ }^{3} \mathrm{~A}_{2 q} \rightarrow{ }^{3} \mathrm{~T}_{2}$ $13000 \mathrm{~cm}^{-1}-7000 \mathrm{~cm}^{-1},{ }^{3} \mathrm{~A}_{29} \rightarrow{ }^{3} \mathrm{~T}_{1 \mathrm{~g}}$ (F) $20000 \mathrm{~cm}^{-2}-10000 \mathrm{~cm}^{-1}$ and ${ }^{3} \mathrm{~A}_{2 g} \rightarrow$ ${ }^{3} \mathrm{~T}_{1 \mathrm{~g}}$ (P) $28000 \mathrm{~cm}^{-1}-2000 \mathrm{~cm}^{-1}$. For tetrahedral $\mathrm{Ni}$ (II) complexes the transition and bands are ${ }^{3} \mathrm{~T}_{1}(\mathrm{~F}) \rightarrow{ }^{3} \mathrm{~T}_{1}(\mathrm{P}) 15000 \mathrm{~cm}^{-1},{ }^{3} \mathrm{~T}_{1} \rightarrow{ }^{3} \mathrm{~A}_{2} 7000 \mathrm{~cm}^{-1}$ and ${ }^{3} \mathrm{~T}_{1} \rightarrow$ ${ }^{3} \mathrm{~T}_{2}$ transition is not frequently observed. In this present study the benzioc acid Nickel (II) chloride complex shows absorption at $25000 \mathrm{~cm}^{-1}$ which suggest that the Nickel ion is in the octahedral environment.

For copper (II) complexes Jahn-Teller distortion is observed. Because of the operation of Jahn-Teller effect on the ${ }^{2} e_{g}$ ground state octahedral distortion is observed. In the distorted versions several absorption bands may be expected in the region $13000 \mathrm{~cm}^{-1}$ to $18000 \mathrm{~cm}^{-1}$. For tetrahedral complexes of copper (II) a band around $10000 \mathrm{~cm}^{-1}$ is expected. ${ }^{10}$ The syntesised copper (II) chloride complex of benzoic acid has its absorption at $14900 \mathrm{~cm}^{-1}$, suggesting octahedral geometry.

\section{Conclusion}

From the investigation, it is concluded that benzoic acid acts as a mono dentate ligand and forms octahedral complexes with metal chlorides. 


\section{References}

1. Cotton F.A and Wilkinson G. (1980) Advanced Inorganic chemistry IV edition, New York Wiley inter science, Page 1344.

2. Colton F.A and Wilkinson G. (1980) Advanced Inorganic chemistry IV edition, New York Wiley inter science, Page 1340.

3. Bernard L.Oser "Hawks" Physiological Chemistry 14" edition TATA Mc Graw Hill Publishing company Ltd., Page 565.

4. Lawrence D.R. and Bennett P.N. Clinical Pharmacology V edition, ELBS and Churchill Livingstone. Page 417:- 418 .

5. Bernard L. Oser "Hawks" Physiological Chemistry 14 $14^{\text {th }}$ edition TATA Mc Graw Hill Publishing company Ltd., Page 559.

6. Schwar Zunbach G. (1960). Complexometric titrations, New York.

7. : Ploquin (1951) J. Compt. Rend., 233, 162.

8. Vandor W. and Hannik K.M. Signth, Reaction in Inorganic Met. Org. chemistry (II) (1981) (l), 15.

9. Jorgensonn C.K. "Absorption spectra and chemical bonding in complexes", (1962) Oxford, Pegamon Press.

10. Holm R.H., Evevett G.W. Jr., Chakrovarthy (1966) A., Prog. Inorg. Chem. Ed. F.A. Cotton Interscience Publication, Vol. 7, Page 3. 